\title{
COSMIC EVOLUTION IN GENERALISED BRANS-DICKE THEORY
}

\author{
B.K.Sahoo ${ }^{1}$ and L.P.Singh ${ }^{2}$ \\ Department of Physics, \\ Utkal University,Bhubaneswar-751004,India \\ 1 bijaya@iopb.res.in \\ 2 lambodar@iopb.res.in
}

\begin{abstract}
We have studied the Generalised Brans-Dicke theory and obtained exact solutions of $\mathrm{a}(\mathrm{t}), \phi(t)$, and $\omega(t)$ for different epochs of the cosmic evolution. We discuss how inflation, decceleration,cosmic acceleration can result from this solution. The time variation of $G(t)$ is also examined.
\end{abstract}

PACS NO:98.80.-K,98.80.cq

Key words:Generalised Brans-Dicke theory,cosmic evolution,deccelerated and accelerated expansion, inflation, time variation of $\mathrm{G}(\mathrm{t})$. 


\section{INTRODUCTION}

The Brans-Dicke Theory(BD) is defined by a scalar field $\phi$ and a constant coupling function $\omega[1]$.This is perhaps the most natural extension of general theory of relativity (GR) which is obtained in the limit of $\omega \rightarrow \infty$ and $\phi=$ constant[2].This theory interestingly appears naturally in supergravity theory,Kaluza-Klein theories and in all the known effective string actions. One important property of BD theory is that it gives simple expanding solutions[ 3 ]for field $\phi(t)$ and scale factor $a(t)$ which are compatible with solar system experiments[4].The Generalised Brans-Dicke theory(GBD)[2],sometimes referred to as graviton-dilaton or scalar -tensor(ST) theory, is instead, defined by $\omega$ which is an arbitrary function of the scalar field $\phi$ (dilaton) and is thus implicitly a function of time $\omega(\phi(t))$. Hence, it includes a number of models,for every function $\omega$.Naturally,a few attempts have been taken to study the dynamics of the universe using this formalism. Some of the cosmological problems studied under this formalism includes nonsingular universe[5], amplification of gravitational wave[6],solutions for different epochs of cosmic evolution and time variation of Newton's gravitational constant G[7] etc. These problems are studied for a specific forms of $\omega(\phi)$ involving quite complicated time-dependence for a $(\mathrm{t}), \phi(t)$. Thus, it is difficult to draw a clear physical picture about the evolution of the universe.

In order to carry out a detailed study of the dynamics of the cosmic evolution in this formalism,knowledge about exact time-dependence of a $(\mathrm{t}), \phi(t)$ and $\omega(t)$ for different epochs is essential. In a previous work[8],we have obtained power law time-dependence of $\omega(t)$ with the power of time determined in terms of the exponent of scale factor a(t) which is taken to vary as $a(t)=a_{0}\left(\frac{t}{t_{o}}\right)^{\alpha}$. To fix the value $\alpha$ we had taken the help of observational information.Similar result is also arrived at by Diaz-Rivera and Pimentel [9].However, in these works the value of $\alpha$ can not be fixed purely by way of solving the gravitational and scalar field equations. In the present work we take our previous attempt a little further and explore to see if a consistent solution of the GBD equations of motion based on simple power-law temporal behaviour of a, $\phi$ and $\omega$ can fix the time exponents.

We have arranged this paper in the following manner.In the section 2 we obtain the solutions for a, $\phi, \omega$ for different epochs. We present a discussion about the present value of $\omega$ in section 3 and examined the time variation of Newton's gravitational constant G in section 4 and conclude with section 5 . 


\section{FIELD EQUATIONS AND SOLUTIONS}

For a universe filled with perfect fluid and described by Friedman- RobertsonWalker space-time with scale factor $a(t)$ with spatial curvature index $\mathrm{k}$, the gravitational field equations in GBD theory are

$$
\begin{gathered}
\frac{\dot{a}^{2}+k}{a^{2}}+\frac{\dot{a} \dot{\phi}}{a \phi}-\frac{\omega \dot{\phi}^{2}}{6 \phi^{2}}=\frac{\rho}{3 \phi} . \\
2 \frac{\ddot{a}}{a}+\frac{\dot{a}^{2}+k}{a^{2}}+\frac{\omega \dot{\phi}^{2}}{2 \phi^{2}}+2 \frac{\dot{a} \dot{\phi}}{a \phi}+\frac{\ddot{\phi}}{\phi}=-\frac{P}{\phi} .
\end{gathered}
$$

where $\rho$ and $\mathrm{P}$ are respectively the energy density and pressure of the fluid distribution. The equation of state of the fluid is given by $P=\gamma \rho$.Some of the values of $\gamma$ for typical cases are -1 (vacuum),0(dust),1/3(radiation),1(massless scalar field). The wave equation for scalar field in the GBD theory is [2]

$$
\ddot{\phi}+3 \frac{\dot{a} \dot{\phi}}{a}=\frac{\rho-3 P}{2 \omega+3}-\frac{\dot{\omega} \dot{\phi}}{2 \omega+3} .
$$

Energy conservation equation, which can be obtained from eqs.(1),(2) and (3) is,

$$
\dot{\rho}+3 \frac{\dot{a}}{a}(\rho+P)=0 .
$$

To obtain a cosistent solution for the equations of motion we assume simple power law dependence on time for a, $\phi$ and $\omega$ in the following form which provides simple expanding solutions[6-10],

$$
\begin{gathered}
a=a_{0}\left(\frac{t}{t_{0}}\right)^{\alpha} . \\
\phi=\phi_{0}\left(\frac{t}{t_{0}}\right)^{\beta} . \\
\omega(\phi(t)) \approx \phi^{n}=\omega_{0}\left(\frac{t}{t_{0}}\right)^{n \beta} .
\end{gathered}
$$

$\alpha$ is a positive number to ensure an expanding universe and $n$ is a non zero constant. $\alpha, \beta$ and $n$ are the free parametres at this level whose values we 
wish to fix by way of obtaining consistent solutions of the gravitational and scalar field equations of motion. $a_{0}, \phi_{0}$ are present values of $\mathrm{a}(\mathrm{t})$ and $\phi(t)$.We further assume $k=0$ to remain consistent with inflationary paradigm, which has received strong observational support.Before proceeding further,it may be noted that eqn(4) has the solution,

$$
\rho=C a^{-3(1+\gamma)}
$$

and so

$$
\rho=\rho_{0}\left(\frac{t}{t_{0}}\right)^{-3 \alpha(1+\gamma)}
$$

$\rho_{0}=C a_{0}$ is the $\operatorname{present}\left(t=t_{0}\right)$ value of energy density.

We now discuss the solutions of the field equations in different epochs.

\subsection{Radiation dominated epoch: $\gamma=1 / 3$}

For radiation dominated epoch the gravitational field equation [eqn.(1)] and scalar field wave equation [eqn.(3)] for $\mathrm{k}=0$ become

$$
\left(\frac{\dot{a}}{a}\right)^{2}=\frac{\rho}{3 \phi}-\frac{\dot{a} \dot{\phi}}{a \phi}+\frac{\omega}{6}\left(\frac{\dot{\phi}}{\phi}\right)^{2} .
$$

and

$$
\ddot{\phi}+3 \frac{\dot{a}}{a} \dot{\phi}+\frac{\dot{\omega} \dot{\phi}}{2 \omega+3}=0 .
$$

Using equation (5),(6),(7) and (8), eqn.(9) yields

$$
\left(\frac{\alpha}{t}\right)^{2}=\frac{\rho_{0}}{3 \phi_{0}}\left(\frac{t}{t_{0}}\right)^{-4 \alpha-\beta}-\frac{\alpha \beta}{t^{2}}+\frac{\omega_{0}}{6}\left(\frac{t}{t_{0}}\right)^{n \beta} \frac{\beta^{2}}{t^{2}}
$$

This equation can be put in the form,

$$
\left(\alpha^{2}+\alpha \beta\right)-\frac{\rho_{0} t_{0}^{2}}{3 \phi_{0}}\left(\frac{t}{t_{0}}\right)^{-4 \alpha-\beta+2}=\frac{\omega_{0} \beta^{2}}{6}\left(\frac{t}{t_{0}}\right)^{n \beta}
$$

This equation is satisfied when

$$
\begin{gathered}
-4 \alpha-\beta+2=n \beta \\
\alpha^{2}+\alpha \beta=0 \\
\beta^{2}=-\frac{2 \rho_{0} t_{0}^{2}}{\omega_{0} \phi_{0}} .
\end{gathered}
$$


Eqn.(10), in similar manner leads to

$$
\beta=0
$$

and

$$
\beta=\frac{(1-3 \alpha)\left(2 \omega_{0}+3\right)}{(n+2) \omega_{0}+3}
$$

Here we have further assumed

$$
\Omega(t)=2 \omega(t)+3=\Omega_{0}\left(\frac{t}{t_{0}}\right)^{n \beta}, \text { where } \Omega_{0}=2 \omega_{0}+3 .
$$

Power law solutions (5),(6),(7) satisfy eqns.(9)and (10) simultaneously when eqn.(11)and eqn.(12) are simultaneously satisfied with $\beta$ given by either eqn,(14) or eqn.(15).

The $\beta=0$ solution of eqn.(14) implies $\alpha=1 / 2$ (from eqn.(11)) and $\phi=$ constant and $\omega=$ constant which represents the well known Standard Model solutions.Thus, $\beta=0$ solution represents the GR sector of GBD theory.

Now using eqn.(15) in eqn.(11)one gets

$$
\alpha=\frac{3 n-2 \omega_{0}-3}{2 n \omega_{0}-2 \omega_{0}+9 n-3}
$$

Similarly,eqn.(15)in conjunction with eqn.(12) gives

$$
\alpha=\frac{2 \omega_{0}+3}{(4-n) \omega_{0}+6}
$$

For eqns(11),(12) and (15) to be simultaneously satisfied, above two values of $\alpha$ should be equal. Imposition of this condition leads to

$$
3 \omega_{0} n^{2}+\left(2 \omega_{0}^{2}+9 \omega_{0}+9\right) n+\left(6 \omega_{0}^{2}+15 \omega_{0}+9\right)=0
$$

which admits two solutions like, $n=-\frac{\left(2 \omega_{0}+3\right)}{\omega_{0}}$ and $-\frac{\left(2 \omega_{0}+3\right)}{3}$.

Substitution of these two values of $\mathrm{n}$ in eqn.(17), leads to $\alpha=1 / 3$ and $\alpha=\frac{3}{\omega_{0}+6}$ respectively. Once again we find from eqn.(15)for $\alpha=1 / 3, \beta$ is equal to zero implying constant $\phi$ and constant $\omega$. We neglect this solution as it does not capture the characteristics of BD theory.Now for the second solution $\alpha=\frac{3}{\omega_{0}+6}$ we find $\beta=-3 /\left(\omega_{0}+6\right)$ and $n \beta=\frac{2 \omega_{0}+3}{\omega_{0}+6}$. Hence, the time dependences are obtained as

$$
a(t)=a_{0}\left(\frac{t}{t_{0}}\right)^{\frac{3}{\omega_{0}+6}}
$$




$$
\begin{aligned}
& \phi(t)=\phi_{0}\left(\frac{t}{t_{0}}\right)^{-\frac{3}{\omega_{0}+6}} \\
& \omega(t)=\omega_{0}\left(\frac{t}{t_{0}}\right)^{\frac{2 \omega_{0}+3}{\omega_{0}+6}}
\end{aligned}
$$

As can be seen from above, the time dependence of $a(t) \phi(t)$ and $\omega(t)$ are completely fixed once $\omega_{0}$ value is known. $\omega_{0}$ needs to be fixed from other physical considerations. One can recover the standard model result for $a(t)$ from above equations when $\omega_{0}=0$.Further it is easily seen that for ensuring deccelerated expansion in the radiation $\operatorname{epoch}(0<\alpha<1) \omega_{0}$ should be greater than -3 .The values for $\beta$ and $n \beta$ are obtained to lie in the range $-1<\beta<0$ and $-1<n \beta<2$ respectively.

\subsection{Matter dominated epoch: $\gamma=0$}

Using eqn.(5),(6) and(7), in eqn(1) we get

$$
\left(\frac{\alpha}{t}\right)^{2}=1 / 3 \frac{1}{\phi_{0}}\left(\frac{t}{t_{0}}\right)^{-\beta} \rho_{0}\left(\frac{t}{t_{0}}\right)^{-3 \alpha}-\frac{\alpha \beta}{t^{2}}+\frac{\omega_{0}}{6}\left(\frac{t}{t_{0}}\right)^{n \beta}\left(\frac{\beta}{t}\right)^{2},
$$

which can be written in the form

$$
-\frac{\rho_{0} t_{0}^{2}}{3 \phi_{0}}\left(\frac{t}{t_{0}}\right)^{2-3 \alpha-\beta}+\left(\alpha^{2}+\alpha \beta\right)=\frac{\beta^{2} \omega_{0}}{6}\left(\frac{t}{t_{0}}\right)^{n \beta} .
$$

This equation is satisfied when

$$
\begin{gathered}
2-3 \alpha-\beta=n \beta \\
\alpha^{2}+\alpha \beta=0 \\
\beta^{2}=-2 \frac{\rho_{0} t_{0}^{2}}{\phi_{0} \omega_{0}}
\end{gathered}
$$

Similarly,using eqn.(5),(6),(7) in eqn.(3) one gets

$$
\left(\beta(\beta-1)+3 \alpha \beta+\frac{n \beta^{2} \omega_{0}}{\Omega_{0}}\right) \frac{\phi_{0}}{t_{0}^{2}}\left(\frac{t}{t_{0}}\right)^{\beta-2}=\frac{\rho_{0}}{\Omega_{0}}\left(\frac{t}{t_{0}}\right)^{-3 \alpha-n \beta} .
$$

This equation is satisfied when

$$
\beta-2=-3 \alpha-n \beta
$$


and

$$
\left(\beta(\beta-1)+3 \alpha \beta+\frac{n \beta^{2} \omega_{0}}{\Omega_{0}}\right) \frac{\phi_{0}}{t_{0}^{2}}=\frac{\rho_{0}}{\Omega_{0}},
$$

With the help of eqn.(20),eqn.(22) reduces to

$$
\left(1+\frac{n \omega_{0}}{2 \omega_{0}+3}+\frac{\omega_{0}}{2\left(2 \omega_{0}+3\right)}\right) \beta^{2}+(3 \alpha-1) \beta=0 .
$$

The above equation leads to, $\beta=0$ and

$$
\beta=\frac{(1-3 \alpha)\left(4 \omega_{0}+6\right)}{(5+2 n) \omega_{0}+6}
$$

Inserting $\beta=0$,eqn.(21) leads to $\alpha=2 / 3$. This is the standard model result representing GR sector of GBD theory. Using eqn.(23), eqn.(19) leads to

Also for eqn.(23),eqn.(18) leads to

$$
\alpha=\frac{4 \omega_{0}+6}{(7-2 n) \omega_{0}+12}
$$

$$
\alpha=\frac{(n+1)\left(4 \omega_{0}+6\right)-2\left[5 \omega_{0}+2 \omega_{0} n+6\right]}{3\left[(n+1)\left(4 \omega_{0}+6\right)-\left(5 \omega_{0}+2 \omega_{0} n+6\right)\right]}
$$

For consistent solution of field equations, these two values of $\alpha$ should be equal.And we find

$$
2 \omega_{0} n^{2}+\left[2 \omega_{0}^{2}+9 \omega_{0}+6\right]+\left[5 \omega_{0}^{2}+16 \omega_{0}+12\right]=0 .
$$

Which admits two solutions like, $n=-\frac{\left(5 \omega_{0}+6\right)}{2 \omega_{0}}$ and,$n=-\left(\omega_{0}+2\right)$.

The first value of $\mathrm{n}$ is neglected as it once again gives $\alpha=\frac{1}{3}$ and $\beta=0$ as in radiation dominated case. We ignore this solutions since it does not capture the time-varying characteristic of $\phi(t)$ and $\omega(t)$ specific to the GBD theory.

The second value of $n$ leads to $\alpha=\frac{2}{\omega_{0}+4}$.

Hence,the relevant solutions in the matter-dominated epoch are,

$$
\begin{gathered}
a(t)=a_{0}\left(\frac{t}{t_{0}}\right)^{\frac{2}{\omega_{0}+4}} \\
\phi(t)=\phi_{0}\left(\frac{t}{t_{0}}\right)^{-\frac{2}{\omega_{0}+4}} \\
\omega(t)=\omega_{0}\left(\frac{t}{t_{0}}\right)^{\frac{2\left(\omega_{0}+2\right)}{\omega_{0}+4}}
\end{gathered}
$$

Once again it is found that the time-dependences of $a(t), \phi(t)$, and $\omega(t)$ are 
entirely controlled by $\omega_{0}$, the present value of $\omega$ as was also the case in the radiation-dominated epoch.For deccelerated cosmic expansion $\omega_{0}$ need be greater than -2 . That way even very large values of $\omega_{0}$ required for agreement with solar system measurements[2] can also accomodated.The presently observed accelerated expansion too can be accomodated if $-4<\omega_{0}<$ -2 .The values of $\beta$ and $n \beta$ are found to lie in the range $-1<\beta<0$ and $0<n \beta<2$ respectively.

\subsection{Vacuum dominated epoch: $\gamma=-1$}

Using eqn.(5),(6) and (7),eqn.(1) reduces to

$$
\left(\frac{\alpha}{t}\right)^{2}=\frac{\rho_{0}}{3 \phi_{0}}\left(\frac{t}{t_{0}}\right)^{-\beta}-\frac{\alpha \beta}{t^{2}}+\frac{\omega_{0}}{6}\left(\frac{t}{t_{0}}\right)^{n \beta}\left(\frac{\beta}{t}\right)^{2}
$$

This can be written as

This equation is satisfied when

$$
\left(\alpha^{2}+\alpha \beta\right)-\frac{\beta^{2} \omega_{0}}{6}\left(\frac{t}{t_{0}}\right)^{n \beta}=\frac{\rho_{0} t_{0}^{2}}{3 \phi_{0}}\left(\frac{t}{t_{0}}\right)^{2-\beta}
$$

$$
\begin{gathered}
2-\beta=n \beta \\
\alpha^{2}+\alpha \beta=0 \\
-\frac{\beta^{2} \omega_{0}}{6}=\frac{\rho_{0} t_{0}^{2}}{3 \phi_{0}}
\end{gathered}
$$

Similarly,using eqn.(5),(6),(7),eqn.(3) reduces to

$$
\left(\beta(\beta-1)+3 \alpha \beta+\frac{n \beta^{2} \omega_{0}}{\Omega_{0}}\right) \frac{\phi_{0}}{t_{0}^{2}}\left(\frac{t}{t_{0}}\right)^{\beta-2}=\frac{4 \rho_{0}}{\Omega_{0}}\left(\frac{t}{t_{0}}\right)^{-n \beta}
$$

This equation is satisfied when

$$
2-\beta=n \beta
$$

and

$$
\beta(\beta-1)+3 \alpha \beta+\frac{n \beta^{2} \omega_{0}}{\Omega_{0}}=\frac{4 \rho_{0} t_{0}^{2}}{\left(2 \omega_{0}+3\right) \phi_{0}}
$$

The above equation with the help eqn.(27) reduces to

$$
\left(1+\frac{(n+2) \omega_{0}}{\left(2 \omega_{0}+3\right)}\right) \beta^{2}+(3 \alpha-1) \beta=0
$$


The $\beta=0$ solution of this equation which leads to inconsistency with respect to eqns(25) and (28),we believe, is indicative of the impossibility of obtaining exponential standard model expansion within the power law assumptions considered in this work. Continuing further,equation(28) and (29) give

However,equation(25) and (26)give

$$
\alpha=\frac{3(n-1)-6 \omega_{0}}{3\left(2 \omega_{0}+3\right)(n+1)}
$$

$$
\alpha=-\frac{2}{n+1}
$$

Now these two values of $\alpha$ are equated for consistent solutions of equation(1) and (3).Hence, we get

$$
n=-\left(2 \omega_{0}+5\right)
$$

This value of $\mathrm{n}$ leads to $\alpha=\frac{1}{\omega_{0}+2}$ and $\beta=-\frac{1}{\omega_{0}+2}$

Hence, the exact time dependence of $\mathrm{a}(\mathrm{t}), \phi(t)$ and $\omega(t)$ in case of vacuum energy dominated epoch are obtained as,

$$
\begin{aligned}
& a(t)=a_{0}\left(\frac{t}{t_{0}}\right)^{\frac{1}{\omega_{0}+2}} \\
& \phi(t)=\phi_{0}\left(\frac{t}{t_{0}}\right)^{-\frac{1}{\omega_{0}+2}} \\
& \omega(t)=\omega_{0}\left(\frac{t}{t_{0}}\right)^{\frac{2 \omega_{0}+5}{\omega_{0}+2}}
\end{aligned}
$$

Once again, as in earlier two cases discussed, the time dependence is controlled by $\omega_{0}$, the present value of $\omega$.For accelerated cosmic expansion we must have $-2<\omega_{0}<-1$. In fact,closer the value of $\omega$ to -2 faster is the accleration which can mimic inflation.Such a power-law inflation was indeed obtained by Mathiazhagan and Zohri,and La and Steinhardt[3] in their pioneering works.Further in this epoch we obtain $\beta$ and $n \beta$ values to lie in the range $-\infty<\beta<-1$ and $3<n \beta<\infty$ respectively for above stated range of $\omega_{0}$.

\section{Present value of $\omega(\phi)$}

In order to look for answer to the recently observed accelerated expansion of the universe,structure formation,coincidence problem etc.some authers have 
studied cosmological models in Brans-Dicke theory or some modified version of this theory[10].A small negative value of $\omega$ at $t \rightarrow \infty$ lying in the range $-2<\omega_{0}<-3 / 2$ has been a recurrent result in these studies ,in contrdiction with large values of the order of 600[2] obtained from solar system measurements. A possible solution of this contradiction,we believe, may lie in considering a non-constant coupling function $\omega(\phi)$ as in GBD theory.Thus, the value of such a function can change with the cosmic time and,in the limit $t \rightarrow \infty$, it could agree with local measured values.

Further,as evident from our solutions, the time dependence of $a(t), \phi(t)$ and $\omega(\phi(t))$ in various epochs are entirly controlled by $\omega_{0}$, the present value of $\omega$ and not by the values of $\omega$ during the epochs considered. This brings out the importance of the value of $\omega_{0}$. For our results obtained in this work to be consistent with the results obtained by earlier authers[10], $\omega_{0}$ can safely lie in the range $-2<\omega_{0}<-1$ excluding the $\omega_{0}=-3 / 2$ value for which $\mathrm{BD}$ theory breaks down. Thus, we arrive at a comprehensive picture of cosmic evolution in the important epochs with the possibility of obtaining sufficient inflation in vacuum dominated era as well as accelerated expansion in the present epoch.Given the simple assumptions considered by us, we consider this a very interesting result which signifies the power of a GBD theory.

\section{VARIATION OF NEWTON'S GRAVITA- TIONAL CONSTANT G:}

As is well known, for theories with constant $\omega$, the possibility of variations of $G$ is very small.Consideration of arbitrary coupling function $\omega(\phi)$, however,opens the possibility of variations of G.In the weak field limit Nordtvedt[2] found an expression for the observed value of the gravitational constant

$$
G(t)=\left(\frac{4+2 \omega(\phi)}{3+2 \omega(\phi)}\right) \phi^{-1}
$$

The variation rate of $\mathrm{G}$ is[2]

$$
\frac{\dot{G}}{G}=-\left[\frac{\dot{\phi}}{\phi}+\frac{2 \dot{\omega}}{(3+2 \omega)(4+2 \omega)}\right]
$$


where over dots represent time derivative.

The present variation rate,therefore, is given by

$$
\left(\frac{\dot{G}}{G}\right)_{0}=-\left(\frac{\dot{\phi}}{\phi}\right)_{0}-\frac{2(\dot{\omega})_{0}}{\left(3+2 \omega_{0}\right)\left(4+2 \omega_{0}\right)}
$$

For typical values of $\omega_{0}$ like -1.9 or -1 ,we obtain from the above expression, $\left|\frac{\dot{G}}{G}\right|_{0} \approx 1.3 H_{0} \approx 2 \times 10^{-10} \mathrm{yr}^{-1}$. Thus, the variation of $\mathrm{G}$ is safely below the observational limit, which is $4 \times 10^{-10} \mathrm{yr}^{-1}[10,11]$ obtained within the present formalism.

\section{CONCLUSIONS}

In this work,we have studied a homogeneous and isotropic cosmological model in the context of a generalised scalar-tensor theory. We have solved consistently both the gravitational field equation and scalar field wave equation for matter,radiation and vacuum dominated epochs of the cosmic evolution by assuming power law time-dependence for the scale factor,scalar field and coupling parameter as extension of our earlier work[8]. The solutions are generic which deserves attention.For all epochs, the solutions depend on one parameter i.e,on the present value of $\omega$.Interestingly,general considerations about cosmic evolution can constraint this value to lie in the range $-2<\omega_{0}<-1$.This result is quite consistent with other results[10] obtained from various other considerations.Also, we have shown that, when $\omega_{0}$ is chosen very close to -2 one can produce enough inflation according to wish, which can solve inflationary problems very easily in the standard model. While obtaining usual decceleration when the universe is radiation or matter dominated we also exhibit the possibility of accelerated expansion in matter dominated era for a slightly different value of $\omega_{0}$.

We also find that the B-D scaler field $\phi(t)$ is always a decreasing function of time in all epochs with the decrease becoming very significant in vacuum dominated case. The time dependence of $\omega(t)$, on the contrary,does not exibit such monotonic temporal behaviour.

We have also examined the time variation of $\mathrm{G}(\mathrm{t})$ in this solution and found that the solution gives a safe time variation for the chosen range of $\omega_{0}$ which gives a strong support to the results presented here. 
Acknowldgement

The authors are grateful to DST ,Govt.of India for providing financial support. The authors also thank Institute of Physics,Bhubaneswar,India, for providing facility of the computer center.

\section{References}

[1] C.H.Brans and R.H.Dicke;Phys.Rev.124 (1961) 925.

[2] K.Nordtvedt,Jr.,Astrophys.J.161 (1970) 1059; J.D.Benkestein and A.meisels,Phys.Rev.D 18 (1978) 4378; J.M.Alimi and A.Serna Phys.Rev.D,53 (1996) 3074,Gravitation and Cosmology ,2 (1996) 7.

[3] C.Mathiazhagan and V.B.Johri,Class.Quantum Grav.1 (1984) L29;

D.La and P.J.Steinhardt,Phys.Rev.Lett 62 (1989) 374

[4] S.Perlmutter etal.,Astrophys.J.517 (1999) 565;

A.G.Riess etal,Aston.J.116 (1999) 74;

P.M.Garnavich etal,Astrophys.J.509 (1998) 74.

[5] S.Kalyana Rama and S.Gosh,Phys.Lett.B 383 (1996) 32. S.Kalyana Rama,Phys.Lett.B 373 (1996) 282.S.kalyana Rama,Singularity Free universe in Graviton-Dilaton Models,hep-th/960826.

[6] J.D.Barrow etal. Phy.Rev.D,48 (1993) 3630.

[7] J.D.Barrow,Phys.Rev.D. 47 (1993) 5329.

[8] B.K.Sahoo and L.P.Singh,to appear in Modern Physics Letter A, gr-qc/0210004.

[9] Diaz-Rivera and L.Pimentel,gr-qc/0208061.

[10] O.Bertolami and P.J.Martins, Phys.Rev.D,61 (2000) 064007 ;N.Benerjee and D.Pavon,Phys.Rev.D 63 (2001) 043504;S.Sen and T.R.Seshadri, grqc/0007079.

[11] S.Weinberg,Gravitation and Cosmology ,Wiley,New York,1972 . 